\title{
Introduction to the focused issue on the 2016 ACM/IEEE-CS Joint Conference on Digital Libraries JCDL 2016
}

\author{
Richard Furuta ${ }^{1} \cdot$ Michele C. Weigle $^{2}$
}

Published online: 12 November 2019

(c) Springer-Verlag GmbH Germany, part of Springer Nature 2019

We are pleased in this issue to present extended and enhanced versions of four award-nominated papers from the 2016 ACM/IEEE-CS Joint Conference on Digital Libraries (JCDL '16). JCDL ' 16 was held on the campus of Rutgers University-Newark and is the sixteenth in the series of conferences jointly sponsored by the Association for Computing Machinery and the IEEE Computer Society. The location is particularly notable since it also was one of the homes of one of the two conference series that merged to create JCDL-the IEEECS Advances in Digital Libraries. (The other conference was ACM Digital Libraries.) Thus, JCDL ' 16 reflects both the enormous advancement adoption and that have occurred to date in digital libraries, but also the novel, multidisciplinary, and dynamic research issues that they continue to engender.

JCDL can lay claim to being the preeminent academic conference for digital library research and so the papers in this issue reflect the best of what already is a highly selective group. JCDL papers are submitted in complete and finished form and are evaluated carefully by the conference's program committee. Only $29 \%$ of the full paper submissions were selected for inclusion in the conference. A smaller group of papers were identified from the conference's papers as candidates for one of the conference's two full paper awards-papers selected either were specifically recommended by program committee members and/or were ones receiving the most positive reviews. Those authors were then invited to submit extended versions of their contributions to this journal. These submissions were reviewed again using the normal procedures of the journal, a

Richard Furuta

furuta@cs.tamu.edu

Michele C. Weigle

mweigle@cs.odu.edu

1 Department of Computer Science and Engineering, Texas A\&M University, 3112 TAMU, College Station, TX 77843-3112, USA

2 Old Dominion University, 5115 Hampton Blvd, Norfolk, VA 23529, USA process potentially requiring revision and re-review, and ultimately were accepted for publication.

The four papers that were accepted for inclusion in this issue are:

- "Time-focused Analysis of Connectivity and Popularity of Historical Persons in Wikipedia," by Adam Jatowt, Daisuke Kawai, and Katsumi Tanaka, an extended version of the JCDL '16 paper "Digital History Meets Wikipedia: Analyzing Historical Persons in Wikipedia."

- "Capisco: Low-cost Concept-Based Access to Digital Libraries," by Annika Hinze, David Bainbridge, Sally Jo Cunningham, Craig Taube-Schock, Rangi Matamua, J. Stephen Downie, and Edie Rasmussen, and extended version of the JCDL '16 paper "Low-cost Semantic Enhancement to Digital Library Metadata and Indexing: Simple Yet Effective Strategies."

- "Comparing Published Scientific Journal Articles to their Pre-Print Versions," by Martin Klein, Peter Broadwell, Sharon E. Farb, and Todd Grappone, an extended version of the JCDL '16 paper of the same title, which paper received the JCDL '16 conference's Vannevar Bush Best Paper Award.

- "Assessing the Quality of Answers Autonomously in Community Question-Answering," by Long. T. Le, Chirag Shah, and Erik Choi, an extended version of the JCDL '16 paper "Evaluating the Quality of Educational Answers in Community Question-Answering," which received the JCDL '16 conference's Best Student Paper Award.

We feel that this issue's papers emphasize the diversity and depth behind research into digital libraries. We thank the authors for their contributions, applaud their achievements, and hope that you will find the papers thought-provoking and enjoyable.

Publisher's Note Springer Nature remains neutral with regard to jurisdictional claims in published maps and institutional affiliations. 